\title{
Prevalencia de caries en una población escolar de doce años
}

\section{Prevalence of caries in an school population of twelve year-old}

\author{
Tapias Ledesma MA*, Martín-Pero L**, Hernández $\mathrm{V}^{* * *}$, Jiménez $\mathrm{R}^{* * * * *}$, \\ Gil de Miguel A*****
}

\section{RESUMEN}

El objetivo de este estudio es conocer la prevalencia de caries en una población escolar de 12 años y factores asociados a la misma.

Población y métodos: Se ha efectuado un estudio transversal en 452 escolares de 12 años edad de Móstoles. Para determinar el estado dental se ha efectuado una exploración odontológica siguiendo los criterios de la OMS. Medimos la prevalencia de caries en dentición temporal y permanente, así como los índices de caries en dentición temporal (cod, cos) y en dentición permanente (CAOD, CAOS), también medimos el índice SiC, calcularemos los intervalos de confianza al 95\%. Utilizamos el programa estadístico SPSS V 13 para realizar todos los cálculos estadísticos y epidemiológicos necesarios del estudio.

Resultados: La prevalencia en dentición permanente es del 28,8\% (IC 95\%: 26,94-31,06). El índice CAOD es del 0,61 (IC 95\%: 0,48-0,73) y el índice cod 0,33 (IC 95\%: 0,24-0,42). El índice SiC es de 1,82 (IC 95\%: 1,55-2,09). Como conclusión podemos resaltar que prevalencia de caries en la población de 12 años de Móstoles ha disminuido. Un $71 \%$ de los escolares esta libre de caries en dentición permanente.

Palabras clave: Prevalencia, caries dental, SiC.

\section{SUMMARY}

The objective of study is to know the prevalence of dental caries in schoolchildren 12 year-old and associated factors.

Population and Methods: A cross sectional observational study has been carried out in 542 twelve year old schoolchildren in Móstoles. We conducted a dental exploration using the World Health Organization criteria. We then measure the caries prevalence permanent and deciduas and the dmt, dmts, DMFT, DMFS index and its confidence limits at $95 \%$. We used programs SPPS V.13 to carry out all the statistic calculations of the study. Results: The caries prevalence in permanent teeth is 28,8\% (IC95\%: 26,94-31,06). The DMTF index is 0,61 (IC 95\%: 0,48-0,73) and the dmt index is 0,33 (IC 95\%: 0,24-0,42). SiC index is 1,82 (IC 95\%: 1,55-2,09).

In conclusion, the caries prevalence in the 12 year old population from Mostoles to decreed. $71 \%$ the schoolchildren are caries free.

Key words: Prevalence, dental caries, SiC.

Fecha de recepción: 17 de junio 2008.

Aceptado para publicación: 30 junio 2008.

* Médico Estomatólogo. Centro de Salud El Soto. Móstoles. Madrid.

** Higienista. Centro de Salud El Soto. Móstoles. Madrid.

*** Licenciado en Estadística. Facultad Ciencias de la Salud. Universidad Rey Juan Carlos.

**** Prof. Titular Medicina Preventiva y Salud Pública. Facultad Ciencias de la Salud. Universidad Rey Juan Carlos.

***** Catedrático de Medicina Preventiva y Salud Pública. Facultad Ciencias de la Salud. Universidad Rey Juan Carlos.

Tapias Ledesma MA, Martín-Pero L, Hernández V, Jiménez R, Gil de Miguel A. Prevalencia de caries en una población escolar de 12 años. Av. Odontoestomatol 2009; 25 (4): 185-191. 


\section{INTRODUCCIÓN}

La caries dental es un problema de salud pública debido a su alta prevalencia (1, 2). Sin embargo, debido a los programas preventivos de salud bucodental y al aumento del nivel socioeconómico de la población se ha observado una disminución de la prevalencia de caries en la población infantil $(3,4)$.

Al revisar la prevalencia de caries en dentición permanente, observamos en diversos estudios (5-7) que alrededor del año 1990 se obtuvieron prevalencias del $70 \%$ a la edad de 12 años, descendiendo estas cifras entorno al $40-50 \%$ a finales del siglo XX $(7,8)$. Así que, al igual que otros países europeos la salud bucodental de los escolares españoles ha mejorado en los últimos años. Sin embargo, existe un grupo de población que tiene mayor riesgo de padecer la enfermedad y que se asocia a factores sociodemográficos como es pertenecer a la clase social baja (9).

La Organización Mundial de la Salud (OMS) utiliza el índice significativo de caries ( $\mathrm{SiC}$ ) para evaluar el nivel de enfermedad en el tercio de población que tiene mayores valores en el índice CAOD y escoge para evaluar los niveles de enfermedad de caries a la población de 12 años de edad $(10,11)$.

El objetivo del estudio es conocer la prevalencia de caries y factores asociados a la misma en una población escolar de 12 años.

\section{POBLACIÓN Y MÉTODOS}

Se ha realizado un estudio epidemiológico descriptivo transversal en 452 escolares de Móstoles.

Se ofertó entrar en el estudio a los escolares de 12 años de once colegios que pertenecen a la zona de influencia del Centro de Salud "El Soto".

Los criterios de inclusión son el consentimiento informado de padres y/o tutores, cumplir 12 años (haber nacido en el año 1995) y realizarse la encuesta odontológica.

La revisión odontológica se efectuó durante los meses de Marzo y Abril de 2007.
Para determinar el estado dental se ha efectuado una exploración odontológica siguiendo los criterios de la OMS, con espejo plano $\mathrm{n}^{\circ} 5$ y luz natural (12).

Rellenamos una ficha individual donde anotamos en un odontograma los diferentes estados dentales en dentición temporal y permanente, así como variables sociodemográficas. Determinamos la clase social por la profesión del cabeza de familia según la clasificación abreviada de la Sociedad Española de Epidemiología (13).

Mediremos la prevalencia de caries en dentición temporal y permanente, así como los índices de caries en dentición temporal (cod, cos) y en dentición permanente (CAOD, CAOS), también mediremos el índice $\mathrm{SiC}$, calcularemos los intervalos de confianza al $95 \%$.

Compararemos diferentes estados dentales según las variables sociodemográficas sexo y clase social. Realizaremos una distribución de frecuencia de la población que tiene un índice CAOD $>0$ según variables sociodemográficas, y compararemos sus frecuencias mediante el test estadístico del chi cuadrado de Pearson.

Utilizaremos el programa estadístico SPSS V 13 para realizar todos los cálculos estadísticos y epidemiológicos necesarios del estudio.

\section{RESULTADOS}

De los 452 escolares que entraron en el estudio, fueron excluidos 53 por lo que la población quedó constituida por 399 escolares de los cuales 181 son niñas y 218 son niños.

La prevalencia de caries en dentición temporal es del 15,8\% (IC 95\%: 13,98-17,62) y la prevalencia en dentición permanente es del 28,8\% (IC 95\%: 26,9431,06).

El 55,89\% (IC 95\%: 53,41-58,37) tiene uno o más primeros molares permanentes sellados (Tabla 1).

El índice CAOD es del 0,61 (IC 95\%:0,48-0,73) y el índice cod 0,33 (IC 95\%: 0,24-0,42). El índice SiC es de 1,82 (IC 95\%: 1,55-2,09). 


\begin{tabular}{|l|c|c|c|}
\hline \multicolumn{4}{|c|}{ TABLA 1.- DISTRIBUCIÓN DE LA } \\
POBLACIÓN SEGÚN VARIABLES \\
SOCIODEMOGRÁFICAS, SELLADORES \\
Y CARIES EN DENTICIÓN TEMPORAL Y \\
\multicolumn{3}{|c|}{ PERMANENTE } \\
\hline Variables & Categorías & \multicolumn{2}{c|}{ Frecuencias } \\
\cline { 3 - 4 } & & Absolutas & Relativas \\
\hline Sexo & Niña & 181 & $45,40 \%$ \\
& Niño & 218 & $54,60 \%$ \\
\hline Clase social & Alta & 19 & $4,80 \%$ \\
& Media & 119 & $29,80 \%$ \\
& Baja & 261 & $65,40 \%$ \\
\hline Caries dentición & No & 336 & $84,20 \%$ \\
temporal & Si & 63 & $15,80 \%$ \\
\hline Caries dentición & No & 284 & $71,20 \%$ \\
permanente & Si & 115 & $28,80 \%$ \\
\hline Selladores & No & 176 & $44,11 \%$ \\
& Si & 223 & $55,89 \%$ \\
\hline
\end{tabular}

En la distribución de frecuencias de los selladores según sexo y clase social no se obtienen diferencias estadísticamente significativas (Tabla 3).

En la tabla 4 se encuentra la distribución de frecuencias del tercio de población incluida en el índi-

\begin{tabular}{|l|c|c|}
\hline \multicolumn{3}{|c|}{ TABLA 2.- ÍNDICES DE CARIES } \\
EN DENTICIÓN TEMPORAL Y \\
PERMANENTE \\
\hline Índices & Valor (promedio) & IC $95 \%$ \\
\hline cod & 0,33 & $0,24-0,42$ \\
cos & 0,60 & $0,42-0,78$ \\
CAOD & 0,61 & $0,48-0,73$ \\
CAOS & 0,81 & $0,62-1,01$ \\
SiC & 1,82 & $1,55-2,09$ \\
\hline
\end{tabular}

IC 95\%: Intervalos de confianza al 95\%.

ce $\mathrm{SiC}$, donde 52 escolares tienen un índice CAOD igual a 1 .

La distribución de la población con un índice CAOD $>0$ según sexo y clase social se describe en la tabla 5 y se obtienen diferencias estadísticamente significativas $(p<0,05)$ para la clase social.

\section{DISCUSIÓN}

La mayoría de los 53 escolares excluidos del estudio fueron debido a falta del consentimiento informado y no creemos que puedan sesgar los resultados.

\begin{tabular}{|c|c|c|c|c|c|}
\hline \multicolumn{6}{|c|}{$\begin{array}{l}\text { TABLA 3.- DISTRIBUCIÓN DE FRECUENCIAS DE SELLADORES EN PRIMEROS MOLARES } \\
\text { PERMANENTES SEGÚN SEXO Y CLASE SOCIAL, COMPARACIÓN DE LAS MISMAS }\end{array}$} \\
\hline Variables & Categorías & Selladores & Frec. absolutas & Frec. relativas & $\mathrm{p}$ \\
\hline \multirow[t]{2}{*}{ Sexo } & $\begin{array}{l}\text { Niña } \\
\text { Si }\end{array}$ & $\begin{array}{l}\text { No } \\
105\end{array}$ & $\begin{array}{r}76 \\
58,0 \% \\
\end{array}$ & $42,0 \%$ & N.S \\
\hline & $\begin{array}{l}\text { Niño } \\
\text { Si }\end{array}$ & $\begin{array}{l}\text { No } \\
118\end{array}$ & $\begin{array}{r}100 \\
54,1 \%\end{array}$ & $45,9 \%$ & \\
\hline \multirow[t]{3}{*}{ Clase social } & Alta & $\begin{array}{l}\text { No } \\
\text { Si }\end{array}$ & $\begin{array}{r}10 \\
9\end{array}$ & $\begin{array}{l}52,6 \% \\
47,4 \% \\
\end{array}$ & N.S \\
\hline & Media & $\begin{array}{c}\text { No } \\
\text { Si }\end{array}$ & $\begin{array}{l}55 \\
64 \\
\end{array}$ & $\begin{array}{l}46,6 \% \\
53,8 \% \\
\end{array}$ & \\
\hline & Baja & $\begin{array}{c}\text { No } \\
\text { Si }\end{array}$ & $\begin{array}{l}111 \\
150\end{array}$ & $\begin{array}{l}42,5 \% \\
57,5 \%\end{array}$ & \\
\hline
\end{tabular}

p: significación estadística. N.S.: No significativo. 


\begin{tabular}{|c|c|c|}
\hline \multicolumn{3}{|c|}{$\begin{array}{l}\text { TABLA 4.- DISTRIBUCIÓN DEL ÍNDICE } \\
\text { CAOD EN LA POBLACIÓN QUE } \\
\text { PERTENECE AL ÍNDICE SIGNIFICATIVO } \\
\text { DE CARIES (SIC) }\end{array}$} \\
\hline \multirow[t]{2}{*}{ CAOD } & \multicolumn{2}{|c|}{ Frecuencias } \\
\hline & Absolutas & Relativas \\
\hline 10 & 1 & $0,75 \%$ \\
\hline 8 & 1 & $0,75 \%$ \\
\hline 6 & 1 & $0,75 \%$ \\
\hline 5 & 3 & $2,26 \%$ \\
\hline 4 & 10 & $7,52 \%$ \\
\hline 3 & 17 & $12,78 \%$ \\
\hline 2 & 30 & $22,56 \%$ \\
\hline 1 & 51 & $39,10 \%$ \\
\hline 0 & 18 & $13,53 \%$ \\
\hline
\end{tabular}

La prevalecía de caries en dentición temporal es del $15,8 \%$ menor que en la población de 6 años, esto es debido a la exfoliación fisiológica de la dentición temporal y su recambio por la dentición permanente $(9,14,15)$.

La prevalecía de caries en dentición permanente es del $28,8 \%$ mayor que el $7,2 \%$ de la población de 6 años, confirmando que la prevalencia de caries aumenta según aumenta la edad $(9,16)$.

Al comparar la prevalencia obtenida con respecto a otros estudios en nuestro país, encontramos una prevalencia de un $27,99 \%$ similar a la nuestra, en una población escolar de Lugo (17); una prevalencia del $43,3 \%$ en España; y del 52,8\% en una población de Móstoles de 13,5 años tras siete años y medio de programa preventivo, mayores a nuestra, lo que confirma el declinar de la prevalencia de caries ya que ha transcurrido más de una década con respecto a estos dos últimos estudios $(16,18)$.

El índice CAOD es de 0,61 (IC 95\%: 0,48-0,73) y el índice CAOS de 0,81 (IC 95\%: 0,62-1,01), lo que indica unos niveles bajos de caries, ya que al compararlos con resultados obtenidos en otros estudios, encontramos índices CAOD mayores que el nuestro, como un 2,32 en una población de Madrid; 1,53 en Galicia y un 1,07 en Valencia (19-21).

En países de nuestro entorno encontramos índices CAOD de 0,7 en Alemania; 0,8 en Holanda; 0,7 en Reino Unido y un 0,8 en Irlanda, que son similares al nuestro (22-24).

El índice CAOS obtenido es de 0,81 (IC 95\%:0,62$1,01)$ y al compararlo con el índice CAOD $(0,61)$, observamos que la mayoría de los dientes afectados por caries es de una superficie ya que la diferencia entre estos dos índices es pequeña. Al comparar nuestros resultados con otros estudios encontramos un índice CAOS de 2,09 en la población de Móstoles de trece años y medio $(15,16)$. Corrobora el declinar de las superficies dentales afectadas por caries así como en otros países de nuestro entorno (23-24). Sin embargo, en países como Puerto Rico se obtie-

\begin{tabular}{|c|c|c|c|c|c|c|}
\hline \multicolumn{7}{|c|}{$\begin{array}{l}\text { TABLA 5.- DISTRIBUCIÓN DE LA POBLACIÓN CON ÍNDICE CAOD> 0, SEGÚN SEXO Y } \\
\text { CLASE SOCIAL }\end{array}$} \\
\hline \multirow[t]{3}{*}{ Variables } & \multirow[t]{3}{*}{ Categorías } & \multicolumn{4}{|c|}{ Índice CAOD> 0} & \multirow[t]{3}{*}{$\mathrm{p}$} \\
\hline & & \multicolumn{2}{|c|}{ No } & \multicolumn{2}{|c|}{ Si } & \\
\hline & & Fr. absolutas & Fr. relativas & Fr. absolutas & Fr. relativas & \\
\hline Sexo & $\begin{array}{l}\text { Niña } \\
\text { Niño }\end{array}$ & $\begin{array}{l}124 \\
160\end{array}$ & $\begin{array}{l}68,5 \% \\
73,5 \%\end{array}$ & $\begin{array}{l}57 \\
58\end{array}$ & $\begin{array}{l}31,5 \% \\
26,6 \%\end{array}$ & n.s. \\
\hline Clase social & $\begin{array}{c}\text { Alta } \\
\text { Media } \\
\text { Baja }\end{array}$ & $\begin{array}{r}18 \\
88 \\
173 \\
\end{array}$ & $\begin{array}{l}94,7 \% \\
73,9 \% \\
68,2 \%\end{array}$ & $\begin{array}{r}1 \\
31 \\
83 \\
\end{array}$ & $\begin{array}{r}5,3 \% \\
26,1 \% \\
31,8 \%\end{array}$ & $<0,05$ \\
\hline
\end{tabular}


TABLA 6.- ÍNDICES CAOD Y SIC EN PAÍSES DE NUESTRO ENTORNO Y ESPAÑA A LA EDAD DE 12 AÑOS, SEGÚN AÑO DE REVISIÓN

\begin{tabular}{|l|c|c|c|}
\hline País & CAOD & SiC & Año \\
\hline Francia & 1,2 & 3,2 & 2006 \\
Alemania & 0,7 & 2,1 & 2005 \\
Italia & 1,1 & 3,1 & 2004 \\
Rumania & 2,4 & 6,3 & 2006 \\
Inglaterra & 1,2 & 3,2 & 2001 \\
Suecia & 1,0 & 2,9 & 2005 \\
España (Valencia) & 1,1 & 2,9 & 2004 \\
\hline
\end{tabular}

Fuente: $O M S$

nen índices de caries en dentición permanente muy por encima de los nuestros (25).

La prevalencia de selladores oclusales en del $55,89 \%$ esto indica que más de la mitad de los escolares de 12 años tienen uno o mas primeros molares permanentes sellados. Al comparar con otros estudios observamos una prevalencia solo del $4,3 \%$ en Puerto Rico inferior a la nuestra y del $73 \%$ en Irlanda, esto demuestra la efectividad de los selladores ya que a mayor porcentaje de escolares con selladores oclusales se obtienen menores índices de caries, confirmando la efectividad de los mismos $(26,27)$.

El índice $\mathrm{SiC}$ es de 1,82 (IC 95\%: 1,55-2,09) menor de 3, que es el objetivo de la OMS para el año 2015, confirma la efectividad del programa de Salud Bucodental en Móstoles para el tercio de población más afectada (10).

Al comparar nuestro resultado con respecto a otros países de nuestro entorno (tabla 6), observamos que varían desde un índice $\mathrm{SiC}$ de 2,1 al 6,3, lo que confirma la tendencia a cumplir el objetivo de OMS para el año 2015 de un tener un índice significativo de caries menor de 3, excepto en Rumanía, con un SiC de 6,3 (28). De tal manera, en países como Suecia y Alemania donde la población de 12 años tienen unos índices CAOD de 1,0 y 0,7 el índice $\mathrm{SiC}$ es de 2,9 y 2,1 respectivamente, mayores que el nuestro
$(1,82)$, corrobora la efectividad del programa preventivo de salud bucodental de Móstoles, ya que realizamos revisiones odontológicas en los colegios desde primero de educación primaria y captamos a más del $90 \%$ de los niños, y los screening dentales escolares estimulan la asistencia dental y tienen un gran efecto en la población de nivel socioeconómico bajo, disminuyendo las desigualdades de salud dental (29).

Sin embargo, para reducir aún más los índices de caries deberíamos encaminar nuestras estrategias preventivas a la población emigrante ya que Paredes Gallardo V et al, obtienen unos niveles más altos de caries dental en la población inmigrante en comparación con la población autóctona (30).

Al distribuir la población según el índice CAOD $>0$ según variables sociodemográficas obtenemos significación estadística $(p<0,05)$ para la clase social baja. Debemos orientar nuestras estrategias preventivas hacia la población más desfavorecida, de tal manera, que la captación y las medidas preventivas las podamos aplicar en esta población lo antes posible y conseguir una disminución de los niveles de enfermedad en el grupo de población con mayor riesgo de padecer caries dental (31).

\section{CONCLUSIONES}

La prevalencia de caries en la población de 12 años de Móstoles ha disminuido. Un 71\% de los escolares esta libre de caries en dentición permanente y los índices de caries en dentición permanente CAOD y CAOS son menores de 1.

El índice $\mathrm{SiC}$ es de 1,82, y la población de clase social baja tiene mayor afectación de caries, por lo que tenemos que encaminar nuestras estrategias preventivas hacia la población de mayor riesgo.

\section{BIBLIOGRAFÍA}

1. Domínguez V, Acedo MV, Ortega P, Astasio P, Pérez LA, Calle ME. Prevalencia de caries en una población escolar. Av Odontoestomatol 1990;6: 271-9. 
2. Tapias Ledesma MA, Gil de Miguel A, Domínguez Rojas V. Prevalencia de caries en una población infantil de Móstoles. Av Odontoestomatol 2000; 16:241-50.

3. Tapias Ledesma MA, Gil de Miguel A, Domínguez Rojas A. Incidencia de caries en una población infantil de Móstoles: evaluación de un programa preventivo transcurridos cuatro años y medio. Arch Odontoestomatol prev comun 2000; 16:162-9.

4. Tapias Ledesma MA, Jiménez R, Carrasco Garrido $P$; Gil de Miguel $A$. Influence of sociodemografic variables on dental service utilization and oral health among the children included in the year 2001 Spanish Nacional Health Survey. J Public Health Dent 2005;65:215-20.

5. Alvarez Arenal A, Alvarez Riesgo JA, Peña Lopez JM; Fernandez Vazquez JP. DMFT, dmft and treatment requiriments of schoolcildren in Asturias, Spain. Community Dent Oral Epidemiol 1988;26:166-9.

6. Salas-Wadge MH. Dental caries experience in 7, 12 and 14 year-old children in Andalucia, Spain. Community Dent Health1994;11:135-41.

7. Dolado I, Casanas P, Nebol M, Manau C. The prevalence of caries assocated factors in 12 yearold schoolchildren of Barcelona. Aten Primaria 1996; 18:111-5.

8. Llodra-Calvo JC, Bravo-Perez M, CortesMarticorena FJ. Encuesta de Salud Oral en España 2000. RCOE 2002;7:19-63.

9. Tapias-Ledesma MA, Jiménez-García R, Carrasco-Garrido P, Lamas F, Gil de Miguel A. Evolución de la prevalencia de caries en una población infantil de Móstoles entre 1988 y 1997. Av Odontoestomatol 2005;21:333-9.

10. Bratthall D. Introducing the significant caries index together with a proposal for a new global oral health goal for 12-years-olds. Int Dent J. 2000; 50:378-84.
11. Nishi M, Stjernswärd J, Carlsson P, bratthall D. Caries experience of some countries and areas expressed by the Significant Caries index (SiC index). Community Dent Oral Epidemiol 2002; 30:296-301.

12. World Health Organization. Oral Health Surveys: Basic Methods. $4^{\text {th }}$ ed. Geneva. WHO. 1997.

13. Alvarez A, Alonso J, Domingo A, Regidor E. La medición de la clase social en ciencias de la salud. Informe de un grupo de trabajo de la Sociedad Española de Epidemiología. Colección de informes técnicos. Serie Y. 1995:106-11.

14. Iglesias Parada IM. La salud oral en Atención Primaria: (1). Estudio sobre la caries dental en una muestra de 441 niños de Vigo. Av Odontoestomatol 2002;18:285-95.

15. Tapias MA. Incidencia de caries en una población infantil de Móstoles: estudio de cohortes prospectivas [tesis doctoral]. Facultad de Medicina. Universidad Complutense de Madrid.1999.

16. Tapias MA, Gil de Miguel, Jiménez-Garcia R, Gonzalez A, Domínguez V. Incidence of caries in an infant population in Mostoles, Madrid. Evaluation of a preventive program after 7.5 year of follow-up. Int J Paedriatr Dent 2001;11:440-6.

17. Bravo M. Age period-cohort analysis or use in Spain from 1987 to 1997. And analysis based on the Spanish National Health Interview Surveys. Eur J Oral Sci 2001;109:149-54.

18. Lopez-Rabade XC, Smith-Chamosa E. Evaluación de un programa asistencial de salud oral en una población infantil durante cuatro años, en un centro de atención primaria. Aten Primaria 2006; 37:240-40.

19. Esteban-Gallego F. Prevalencia de caries dental en niños entre 1996-2000. Aten Primaria 2002; 30:412-3.

20. Smyth E, Caamaño F. Factors related to dental health in 12-years-old children: a cross-sectional study in pupils. Gac Sanit 2005;19:113-9. 
21. Almerich Silla JM, Montiel Company JM. Oral health survey of the child population in Valencia Region of Spain (2004). Med Oral Patol Oral Cir Bucal 2006;11:369-81.

22. Pitts NB, Evans DJ, Nugut ZJ, Pine CM. The dental caries experience of 12-year-old children in England and Wales. Study of Community Dentistry in 2000/2001. Community Dent Health 2002; 19:45-53.

23. Sagheri D, Mc Loughlin J, Clarkson JJ. A comparison of dental caries levels in two communities with different oral health prevention strategies stratified in different social classes. J Public Health Dent 2007;67:1-7.

24. Schulte AC, Momeni A, Pieper K. Caries prevalence in 12-years-old children from Germany. Results of the 2004 National Survey. Community Dent Health;23:197-202.

25. Elias-Boneta AR, Crespo-Kebler K, Gresbolini CC, Toro-Vizcarrondo CE, Psoter WJ. Dental caries prevalence of twelve year old in Puerto Rico. Community Dent Health 2003;20:171-6.

26. Elias-Boneta AR, Herrero RH, Psoter K, ToroVizcarrondo CE, Psoter WJ. The prevalence of pit and fissure sealants among twelve years olds living in Puerto Rico during 1997. PR Health Sci J 2006;25:133-6.

27. ParnellCA, O‘Farrell $M$, Howell F, Hegarty $M$. Evaluation of a community fissure sealants programme in County Meath, Ireland. Community Dent Health 2003;20:146-52.

28. World Health Organization. WHO Oral Healththe CAPP Index. Significant Caries Index (selected countries) [citado 3 de jun. 2008]. Available: http:/ /www.whocollab.od.mah.se/index.

29. Donaldson M, Kinirons $M$. Effectiveness of the school dental screening programme in stimulating dental attendance for children in need of treatment in Northern Ireland. Community Dent Oral Epidemiol 2001;29:143-9.

30. Paredes Gallardo V, Paredes Cencillo C, Mir Plana B. Prevalencia de la caries dental en el niño inmigrante: estudio comparativo con el niño autóctono. An Pediatr 2006;65:337-41.

31. Jiménez R, Tapias-Ledesma MA, Gallardo-Pino C, Carrasco P, Gil de Miguel A. Influence of sociodemografic variables on use of dental services, oral health and oral higiene among Spanish children. Int Dent 2004;54:187-92.

\section{CORRESPONDENCIA}

Dr. Miguel Ángel Tapias Ledesma

Centro de salud El Soto

Avda. Olímpica 38.

28935 Móstoles.

Madrid

Email: miguelangel.tapias@urjc.es 